\title{
Italie : les réformes de l'école en Italie : 2001-2004
}

\section{Teresa Longo}

\section{OpenEdition}

\section{Journals}

Édition électronique

URL : https://journals.openedition.org/ries/1488

DOI : $10.4000 /$ ries. 1488

ISSN : 2261-4265

\section{Éditeur}

France Education international

\section{Édition imprimée}

Date de publication : 1 décembre 2004

Pagination : 14-17

ISBN : $978-2-85420-564-0$

ISSN : 1254-4590

\section{Référence électronique}

Teresa Longo, «Italie : les réformes de l'école en Italie : 2001-2004 », Revue internationale d'éducation de Sèvres [En ligne], 37 | décembre 2004, mis en ligne le 18 novembre 2011, consulté le 09 juillet 2021. URL : http://journals.openedition.org/ries/1488; DOI : https://doi.org/10.4000/ries.1488

Ce document a été généré automatiquement le 9 juillet 2021

(C) Tous droits réservés 


\section{Italie : les réformes de l'école en Italie : 2001-2004}

\section{Teresa Longo}

1 Les réformes du système éducatif de l'actuel gouvernement italien changent profondément les caractéristiques de l'école publique, suscitant de vifs débats dans le pays.

2 En comparaison avec les autres pays européens d'égal niveau économique, l'Italie apparaît comme un pays où subsistent d'importants retards. Jusqu'en 1999, l'Italie se distinguait, dans les comparaisons internationales, par la brève durée de l'obligation scolaire (huit ans), et par les faibles taux de scolarisation dans le secondaire. En effet, le niveau de scolarisation dans l'école secondaire est parmi les plus bas d'Europe: la moyenne européenne des jeunes de 25/34 ans sans diplôme secondaire est de $29 \%$ (Eurostat, 2000) et de $41 \%$ en Italie. En outre, deux tiers des jeunes qui fréquentent l'école secondaire sont inscrits dans les instituts technico-professionnels. Ils ont libre accès à l'université comme les lycéens, mais échouent facilement en première année et contribuent à l'augmentation des taux d'échec à ce niveau (en 2000, $25 \%$ des étudiants de première année abandonnaient les études supérieures et seulement quatre étudiants sur dix réussissaient à obtenir un diplôme supérieur au bout de six années) ${ }^{1}$.

3 Un autre problème de l'école italienne est l'anachronisme de l'école secondaire : de nombreux projets de réforme ayant échoué pendant un demi-siècle, la structure de l'école secondaire supérieure est restée celle de l'époque fasciste. Toutefois, les enseignants et les établissements disposent de marges importantes de liberté dues à la possibilité d'expérimentations pédagogiques (1974) et à la loi sur l'autonomie des établissements (1999-2000). Cette situation, là où les équipes d'enseignants l'ont voulu, a stimulé une importante innovation des contenus et de la didactique et donné des résultats intéressants mais approfondi également les inégalités de l'offre scolaire déjà existantes dans le système national (nord-sud/îles).

4 À la différence de l'école secondaire, l'école primaire a fait l'objet de réformes qui ont amélioré son enseignement : non seulement l'Italie se situe en bonne place à ce niveau dans les comparaisons internationales, (IEA, 2001), mais l'univers de l'école primaire 
italienne est riche d'expériences intéressantes et innovantes qui ont attiré l'attention de nombreux experts internationaux. Depuis 1991, à l'école primaire, deux enseignants sont également responsables de chaque classe. Leur service couvre trois classes et ils se spécialisent par "grands domaines"; ils sont accompagnés par des enseignants "spécialistes» ou «enseignants de laboratoire» (informatiques, langues ou autre). Cette organisation a donné des résultats très intéressants mais, dans une période d'austérité budgétaire, son coût économique a été considéré comme trop important.

Le processus de réforme de l'école obligatoire a commencé en 1999 quand un gouvernement de centre-gauche a augmenté de deux ans la durée de l'école obligatoire et modifié la structure de son déroulement en sept années d'école de base et cinq années de secondaire ${ }^{2}$.

6 En 2001, le gouvernement de Silvio Berlusconi lance le slogan de l'école des trois «I»: «Impresa, Inglese, Informatica» (Entreprise, Anglais, Informatique) et nomme comme ministre de l'Éducation Letizia Moratti, une femme ayant un profil original par rapport à la tradition du gouvernement pour l'éducation ${ }^{3}$. Comme l'annnonce la ministre dans son premier discours, son programme sera basé sur les principes de "solidarité, d'excellence et d'efficacité », qui ne peuvent se réaliser sans « la lutte quant au poids de l'État dans l'instruction (...). L'État ne peut pas être le seul promoteur de la valeur du capital humain (...). Il doit garantir les principes d'égalité et d'équité en ayant un rôle de contrôle et d'orientation (...) et non de gestion ». Au centre du système éducatif doivent être « la personne » et la famille qui doit avoir « le droit de choisir les parcours éducatifs plus cohérents avec les valeurs individuelles et les objectifs de réalisation personnelle des élèves ». Le système éducatif doit donc devenir un système intégré dans ses composantes publiques et privées, dans la perspective "d'un passage réel de l'école de l'État à l'école de la société civile ${ }^{4}$. La mise en place de la loi du précédent gouvernement sur la réforme des cycles de l'école obligatoire est bloquée, ce qui complique la vie des établissements déjà prêts à mettre en place la prolongation de l'obligation scolaire selon la structure « $7+5 »$. Une nouvelle commission est formée afin d'élaborer des propositions pour la structure, les curricula et la formation des enseignants de tous les niveaux (Commissione Bertagna). Elle doit tenir compte d'un cadre imposé par la ministre selon lequel l'obligation scolaire doit durer douze ans et peut se dérouler également dans le système de formation professionnelle. L'école doit avoir une structure unitaire et le curriculum doit être basé sur des standards nationaux et une flexibilité qui puisse permettre le libre choix des familles.

7 La commission devrait également tenir compte des résultats des états généraux de l'école qui ont rassemblé, fin 2001, plus de mille représentants du monde de l'éducation.

8 Une attention particulière est accordée à l'école privée et à l'enseignement de la religion dans l'école publique. Trente millions d'euros en 2003 (et soixante-dix millions en 2005) sont attribués aux familles qui ont inscrit leurs enfants à l'école privée (en majorité catholique). En ce qui concerne l'organisation de l'éducation catholique, le conseil des ministres approuve, début 2002, un projet de loi portant sur la carrière des enseignants de la religion catholique dans le public. Sur la base d'une liste décidée par l'autorité religieuse (qui évalue les connaissances dans la matière) et d'un concours qui évalue la culture générale et les compétences pédagogiques, l'enseignant devient titulaire d'un contrat à durée déterminée. S'il reste sans poste, il peut être affecté à un autre enseignement pour lequel il a les qualités requises. Cette procédure donne non 
seulement un pouvoir important à l'autorité religieuse sur le recrutement, mais lui permet indirectement d'avoir des personnels validés par elle en situation d'enseigner aussi d'autres disciplines (dans la plupart des cas, la philosophie).

Les travaux de la commission Bertagna constituent la base pour la rédaction d'une loi approuvée en mars 2003 qui stipule que le droit (et non plus l'obligation) à l'instruction et à la formation est assuré pour une durée de douze ans dans une structure de cinq ans d'école primaire, de trois ans d'école secondaire inférieure et de quatre ans d'école secondaire supérieure; l'enseignement professionnel est intégré au système scolaire. La possibilité d'instruction-formation en alternance est aussi prévue. La liberté du choix de l'instruction est garantie par des "plans d'étude personnalisés " constitués d'une partie fondamentale qui reflète la culture nationale et d'une partie optionnelle. L'âge d'accès à l'école maternelle et primaire, si la famille le désire, peut être abaissé d'un an.

10 Dans ce cadre, la loi donne le pouvoir au gouvernement de finaliser la réforme. Un an après, la ministre, par décret ${ }^{5}$, réorganise l'école maternelle et primaire et définit les curricula.

11 Les changements sont importants : l'horaire, défini annuellement, comporte une partie d'enseignements communs et une partie facultative (10\%) organisée par l'école. Pour les activités facultatives, l'école peut embaucher sur ses fonds des experts à contrat à durée déterminée*. Globalement l'horaire diminue et le "temps plein », conquête des mouvements de femmes et des mouvements pour la démocratisation de l'école, n'est possible que si l'école a les ressources pour intégrer le temps financé par l'administration centrale. Le ratio enseignant-élève à l'école primaire change: un enseignant "tutor» sera le responsable de la classe où il enseignera 18/20 heures; les autres heures reviendront à des enseignants qui circuleront entre plusieurs classes. Cette organisation révolutionne le système précédent basé sur l'idée de la responsabilité pédagogique collective de l'équipe des enseignants. Le refus de ce changement par les enseignants du primaire est donc aujourd'hui très fort ainsi que le refus d'une hiérarchisation de leurs rôles professionnels et des disciplines enseignées.

Chez les enseignants, des doutes portent aussi sur l'introduction, à partir de l'école maternelle, du "portfolio de compétences ", un carnet pour chaque élève. Le risque d'un « étiquetage » précoce qui bloque le processus d'instruction est évoqué.

La réforme des curricula fait à présent l'objet d'analyses de la part des associations des enseignants et des écoles; elle introduit des changements culturels et pédagogiques importants. Conçu selon le principe de la flexibilité et du libre choix, le texte du ministère propose les objectifs de la formation, les standards et les critères d'évaluation. Il propose la liste des disciplines de base avec un minimum et un maximum horaire pour chacune. Les programmes indiquent contenus et objectifs à atteindre.

14 L'intérêt de l'opinion publique a été attiré surtout par les programmes de sciences naturelles dans lesquels le sujet de l'évolution «disparaît». Darwin est-il banni des écoles italiennes? Non, a dit la ministre dans une émission télévisuelle mais il ne figure pas dans les programmes. Par contre, le problème «des origines" et du rapport de l'homme à l'environnement est profondément présent dans les programmes de religion catholique. Ici le problème est posé en tant que « réponse aux grands questionnements de la vie » et dans l'espace donné au rapport entre science et religion. Une séparation semble être établie entre l'éducation des premières années où la religion et «les 
réponses élaborées par les familles » sont prioritaires et l'instruction des classes de fin d'études où la science peut être librement apprise.

Le changement pour les enseignants, les familles et les élèves peut donc être important. Il s'agit d'un changement de culture, de rapport à l'école et surtout de philosophie : avant la réforme, surtout à l'école primaire, l'enfant était au centre de l'enseignement et le projet pédagogique de la classe (avec les mêmes objectifs pour tous) prenait en compte la spécificité de chacun. Dans la réforme, l'idée de « personnalisation » domine ; les parcours doivent être construits pour chaque enfant considéré comme partie d'une entité familiale et par chaque établissement scolaire selon le type de public et la volonté des familles.

Pour les adversaires de cette réforme, ce sont les notions mêmes d'individu et de démocratie qui sont ainsi redéfinies.

\section{NOTES}

1. Données de l'ISTAT-MURST 2000-01.

2. Loi n.9 du 20/1/99 et Loi sur les cycles scolaires : 2/2- 2000).

3. Issue d'une famille d'entrepreneurs, $\mathrm{M}^{\mathrm{me}}$ Moratti avait, avant de devenir ministre, été administrateur délégué de la News Corp Europe, société du groupe Murdoch. Présidente de Syntek Capital Group, elle en est encore l'actionnaire la plus importante et Chairman de l'Advisory Board. Enfin, elle a dirigé la RAI ${ }^{3}$.

4. Discours de Letizia Moratti à la Chambre des députés du 18 luglio 2001).

5. DM 19/2/04, N.59.

\section{INDEX}

Mots-clés : réforme de l'enseignement, système éducatif

Index géographique : Italie

\section{AUTEUR}

\section{TERESA LONGO}

Université Jules Verne de Picardie 